\title{
A Secure and Efficient Conference Key Distribution System
}

\author{
(Extended Abstract)
}

\author{
Mike Burmester $^{1 \star}$ and Yvo Desmedt ${ }^{2 \star \star}$ \\ 1 Department of Mathematics, RH - University of London, Egham, Surrey \\ TW20 OEX, U.K., e-mail uhah205@vax.rhbnc.ac.uk \\ ${ }^{2}$ Department of EE \& CS, University of Wisconsin - Milwaukee, P.O. Box 784, \\ Milwaukee WI 53201, U.S.A., e-mail desmedt@cs.uwm.edu
}

\begin{abstract}
We present practical conference key distribution systems based on public keys, which authenticate the users and which are 'proven' secure provided the Diffie-Hellman problem is intractable. A certain number of interactions is needed but the overall cost is low. There is a complexity tradeoff. Depending on the network used, we either have a constant (in the number of conference participants) number of rounds (exchanges) or a constant communication and computation overhead. Our technique for authentication can be extended and used as the basis for an authentication scheme which is 'proven' secure against any type of attack, provided the Discrete Logarithm problem is intractable.
\end{abstract}

\section{Introduction}

To communicate securely over insecure channels it is essential that secret keys are distributed securely. Even if the encryption algorithm used is computationally infeasible to break, the entire system is vulnerable if the keys are not securely distributed. Key distribution is central to cryptography and has attracted a lot of attention (e.g., $[17,24,6,5,30,26,31])$. Research has focused on security and on efficiency. Many practical systems have been proposed [30, 26, 31, 36, 18, 38]. The most familiar system is the Diffie-Hellman key distribution system [17]. This enables two users to compute a common key from a secret key and publicly exchanged information. If more than two users want to compute a common key, then a conference key distribution system is used. Designing such systems can be particularly challenging because of the complexity of the interactions between the many users. Many conference key distribution systems have been presented recently $[24,25,31,36,19,8]$. These however are either impractical, or only heuristic arguments are used to address their security. Our goal in this paper is to present a practical and proven secure conference key distribution system.

Ingemarsson, Tang and Wong proposed several conference key distribution systems in which the common key is a symmetric function [24]. These have many

\footnotetext{
* Research partly carried out while visiting the University of Wisconsin - Milwaukee. * Research partly carried out while visiting Royal Holloway, University of London. Supported in part by NSF Grant NCR-9106327 and NSF Grant INT-9123464.
} 
attractive features, particularly the second order system which has a low communication and computation overhead. However they demonstrated that this particular system is insecure because the information exchanged by the users makes it possible for a passive eavesdropper to compute the key. Our main system is similar, but we use cyclic functions. This prevents the attack by passive eavesdroppers whilst retaining the efficiency of the former scheme. For authentication we use a public key (interactive) authentication scheme which is proven secure assuming the Discrete Logarithm problem is intractable. Combining the two systems we get a conference key distribution scheme which is provably secure against any known type of attack, including those by malicious active adversaries working together, provided the Diffie-Hellman problem is intractable.

Our authentication scheme is of interest in itself, because of its efficiency and proven security. We note that all proven secure signature schemes presented so far $[22,28,33,1,2]$ are impractical. We therefore extend our scheme so that it is proven secure against any type of attack, including adaptive chosen text attacks by real-time middle-persons, under the same cryptographic assumption. The resulting scheme is roughly as fast as RSA [32], but in addition is proven secure.

The organization of this paper is as follows. In Section 2 we give definitions and present our model for conference key distribution systems and for authentication schemes. In Section 3 we present various protocols for conference key distribution systems which are secure against attacks by passive eavesdroppers provided the Diffie-Hellman problem is hard. In Section 4 we adapt the protocols to get authentication. In Section 5 we present an authentication scheme which can be used to get a conference key distribution scheme which is secure against any type of attack. In Section 6 we extend the security of our authentication scheme, and we conclude in Section 7.

Because of page limitations there are no proofs. These will be given in the full version of the paper.

\section{Definitions}

In one of our scenarios we consider networks ${ }^{3}$ in which the users $U_{i}$ can broadcast 'messages' (strings) to each other. We allow for the possibility that an eavesdropper ${ }^{4} E$ (a malicious adversary) may read the broadcast messages or substitute some of them. We distinguish two types of networks: those for which $E$ is passive and those for which $E$ is active. Let $N$ be the security parameter.

${ }^{3}$ A network is a collection of $n$ interactive probabilistic Turing machines $U_{i}$ with $e_{i}^{1}$ write-only tapes, $e_{\imath}^{2}$ read-only tapes, a history tape, a knowledge tape and worktapes.

4 An eavesdropper is an interactive probabilistic Turing machine with $\sum_{i=1}^{n} e_{i}^{1}$ readonly tapes $T_{i j}$ and $\sum_{i=1}^{n} e_{i}^{2}$ write-only tapes $W_{i j}$. The eavesdropper reads from $T_{i j}$ and writes on $W_{i j}$. If what is written is different from what is read then the eavesdropper is active. Otherwise the eavesdropper is passive. This, together with our definition of a network, allows for a scenario in which a broadcasted message can be substituted for each individual receiver. Eavesdroppers are polynomially bounded. 
Definition 1. Suppose that $n=O\left(N^{c}\right), c>0$ constant, interactive Turing machines $U_{1}, \ldots, U_{n}$ take part in a protocol to generate a key. We say that the protocol is a conference key distribution system if, when all the $U_{1}, \ldots, U_{n}$ are as specified, then each $U_{i}$ computes the same key $K=K_{i}$. A conference key distribution system guarantees privacy if it is computationally infeasible for a passive eavesdropper to compute the key $K$.

Definition 2. Suppose that $n=O\left(N^{c}\right)$ interactive Turing machines $U_{1}, \ldots, U_{n}$ use a conference key distribution system, and that each $U_{i}$ has received (from an oracle) a secret key $s_{i}$ (written on its knowledge tape) which corresponds to its public key $k_{i}$, which is published. Let $n^{\prime}>0$ of these be honest ${ }^{5}, n^{\prime \prime}=n-n^{\prime} \geq 0$ be impersonators ${ }^{5}$, and assume that there is a secure network between the impersonators and the (passive or active) eavesdropper. A conference key distribution system is (computationally) secure, if it is computationally infeasible for any set of $n^{\prime \prime}, 0 \leq n^{\prime \prime}<n$, impersonators $U_{j}^{\prime}$ in collaboration with the eavesdropper to compute the same key $K_{i}$ as computed by any of the honest machines $U_{i}$.

Remark. If the set of impersonators is empty then we require that the (active) eavesdropper cannot compute $K_{i}$.

Definition 3. (Informal) Consider a network with eavesdropper $E$. A protocol $\left(U_{1}, U_{2}\right)$ in which $U_{1}$ sends a message $m$ is an authentication system if,

- Compliance: When $U_{1}, U_{2}$ are honest and $E$ is passive then $U_{2}$ accepts and outputs $m$ with overwhelming probability,

- Secure against impersonation: $U_{2}$ rejects with overwhelming probability a dishonest $U_{1}^{\prime}$,

- Secure against substitution: If $E$ is active and $U_{2}$ outputs $m^{\prime} \neq m$ then $U_{2}$ rejects with overwhelming probability.

Definition 4. The Diffie-Hellman [17] problem: given $p, \alpha, \beta, \gamma$, find $\beta^{\log _{\alpha} \gamma} \bmod p$ if it exists.

Breaking this problem has remained an open problem for more than 15 years. Even if the factorization of the order of $\alpha$ is known $[29,15,27,23]$ the problem is assumed to be hard (cf. $[7,9,10])$. It is well known that if the Discrete Logarithm problem is easy then so is the Diffie-Hellman problem, but the converse may not be true.

\section{Private Conference Key Distribution}

In this section we are only concerned with privacy. Authenticity is addressed in Section 5. We consider various conference key distribution systems which are

${ }^{5}$ An honest machine $U_{i}$ has a secret key $s_{i}$ written on its knowledge tape. An impersonator $U_{3}^{\prime}$ is any polynomially bounded interactive probabilistic Turing machine which replaces $U_{3}$ but does not have the secret key of $U_{3}$ (or an equivalent). In our model the eavesdropper is not an impersonator: it can only impersonate $U$, with the help of an impersonator (if there is one). We will strengthen the definition in the final paper to allow for insiders' attacks. 
based on the Diffie-Hellman [17] key exchange. These are designed to exploit the particular configuration of the network used. Our main protocol is in Section 3.3 and Section 3.4. The other protocols are given for comparison.

We use a discrete logarithm setting. A center chooses a prime $p=\Theta\left(2^{N^{c}}\right)$, $c \geq 1$ constant, and an element $\alpha \in Z_{p}$ of order $q=\Theta\left(2^{N}\right)$. If the order has to be verified then the factorization of $q$ is given. The center then publishes $p, \alpha$ and $q$. Let $n$ be polynomially bounded in the length of $p$.

\subsection{A Star Based System}

In this system a chair $U_{1}$ exchanges a Diffie-Hellman key $K_{i}$ with each user $U_{i}$, and then chooses a random conference key $K$ which it sends to each $U_{i}$ encrypted under $K_{i}$. That is,

Protocol 1. Let $U_{1}, \ldots, U_{n}$ be a dynamic set of users ${ }^{6}$ who want to generate a common conference key. $U_{1}$ is the chair.

Step 1 Each $U_{i}, i=1, \ldots, n$, selects ${ }^{7} r_{i} \in R Z_{q}$ and computes $z_{i}=\alpha^{r_{1}} \bmod p$. Then $U_{1}$ sends $z_{1}$ to all the $U_{i}$ and the $U_{i}$ send $z_{i}$ to $U_{1}, i=2, \ldots, n$.

Step $2 U_{1}$ checks $^{8}$ that $\operatorname{ord}(\alpha)=q$. Then $U_{1}$ computes $K_{i}=z_{i}^{r_{1}} \bmod p$ for $i=2, \ldots n$, and selects ${ }^{9}$ a conference session key $K \in_{R}\langle\alpha\rangle . U_{1}$ sends $^{10}$ $Y_{i} \equiv K \cdot K_{i}(\bmod p)$ to each $U_{i}, i=2, \ldots n$.

Step 3 Each $U_{i}, i=2, \ldots, n, \operatorname{checks}^{8}$ that $\operatorname{ord}(\alpha)=q, \operatorname{computes} K_{i} \equiv z_{1}^{r_{i}} \bmod p$, and decrypts $Y_{i}$ to get the session key $K$.

\subsection{A Tree Based System}

This is similar to the star based system, except that a tree configuration network is used. The users $U_{1}, U_{2}, \ldots$ are labelled in such a way that the sons of $U_{a}$ are $U_{2 a}$ and $U_{2 a+1} . U_{1}$ is the root.

Protocol 2. Let $U_{1}, \ldots, U_{n}$ be a dynamic set of users who want to generate a common conference key. $U_{1}$ is the chair.

Step 1 Each $U_{a}$ in the conference selects $r_{a} \in_{R} Z_{q}$ and computes $z_{a}=$ $\alpha^{r_{a}} \bmod p$. Then $U_{a}$ sends $z_{a}$ to $U_{\lfloor a / 2\rfloor}$, if $a>1$, and to $U_{2 a}$ if $2 a \leq n$, and to $U_{2 a+1}$ if $2 a+1 \leq n$.

Step 2 Each $U_{a}$ in the conference $\operatorname{checks}^{8}$ that $\operatorname{ord}(\alpha)=q$. Then if $a>1$ it computes $K_{a}=z_{[a / 2\rfloor}^{r_{a}} \bmod p$ and $K_{2 a+i}=z_{2 a+i}^{r_{a}} \bmod p, i=0,1$, if $2 a+i \leq n . U_{1}$ selects a conference session key $K \in_{R}\langle\alpha\rangle$ and then sends ${ }^{10} Y_{2+i}=K \cdot K_{2+i} \bmod p$ to $U_{2+i}, i=0,1$. Set $\ell=0$.

\footnotetext{
6 Any set of $n$ users, which may dynamically change.

${ }^{7}$ We use the notation $a \in_{R} A$ to indicate that $a$ is selected from the set $A$ uniformly and independently.

8 This check is only done once. If the center is trusted (oracle) it is even not required.

${ }^{9}\langle\alpha\rangle$ is the multiplicative group generated by $\alpha$ in $Z_{p}^{*}$.

${ }^{10}$ Other encryption schemes may be used.
} 
Step $3+\ell$ If $U_{a}$ is at level $\ell$ of the tree (if $\left\lfloor\log _{2} a\right\rfloor=\ell$ ), then $U_{a}$ decrypts $Y_{a}$ to get $K$, and then sends $Y_{2 a+i}=K \cdot K_{2 a+i} \bmod p$ to $U_{2 a+i}, i=0,1$, if $2 a+i \leq n$. Set $\ell:=\ell+1$.

Remark. The users in conference must trust each other against jamming. If $U_{a}$ replaces the key $K$ by $K^{\prime}$ then all his descendents will use $K^{\prime}$, and not $K$.

\subsection{A Broadcast System}

Protocol 3. Let $U_{1}, \ldots, U_{n}$ be a dynamic set of users who want to generate a common conference key. The indices are taken in a cycle: so $U_{n+1}$ is $U_{1}$, and $U_{0}$ is $U_{n}$.

Step 1 Each $U_{i}, i=1, \ldots, n$, selects $r_{i} \in_{R} Z_{q}$, and then computes and broadcasts $z_{i}=\alpha^{r_{i}} \bmod p$.

Step 2 Each $U_{i}, i=1, \ldots, n$, $\operatorname{checks}^{8}$ that $\operatorname{ord}(\alpha)=q$. Then it computes and broadcasts

$$
X_{i} \equiv\left(z_{i+1} / z_{i-1}\right)^{r_{i}}(\bmod p) .
$$

Step 3 Each $U_{i}, i=1, \ldots, n$, computes the conference key,

$$
K_{i} \equiv\left(z_{i-1}\right)^{n r_{2}} \cdot X_{i}^{n-1} \cdot X_{i+1}^{n-2} \cdots X_{i-2}(\bmod p) .
$$

Remark. Honest users compute the same key,

$$
K \equiv \alpha^{r_{1} r_{2}+r_{2} r_{3}+\cdots+r_{n} r_{1}}(\bmod p) .
$$

Indeed, set $A_{i-1} \equiv\left(z_{i-1}\right)^{r_{i}} \equiv \alpha^{r_{i-1} r_{i}}(\bmod p), A_{i} \equiv\left(z_{i-1}\right)^{r_{i}} \cdot X_{i} \equiv \alpha^{r_{i} r_{i+1}}(\bmod p)$, $A_{i+1} \equiv\left(z_{i-1}\right)^{r_{i}} \cdot X_{i} \cdot X_{i+1} \equiv \alpha^{r_{i+1} r_{i+2}}(\bmod p)$, etc., and we have $K_{i}=A_{i-1}$. $A_{i} \cdot A_{i+1} \cdots A_{i-2}$. So the key is a second order cyclic function of the $r_{i}$ (but not symmetric as in [24]).

For $n=2$ we get $X_{1}=X_{2}=1$ and $K \equiv \alpha^{r_{1} r_{2}+r_{2} r_{1}} \equiv \alpha^{2 r_{1} r_{2}}(\bmod p)$, which is essentially the same as for the Diffie-Hellman [17] system (clearly there is no need to broadcast $X_{1}, X_{2}$ in this case).

\subsection{A Cyclic System}

This is similar to the broadcast system except that a bi-directional cyclic network is used. So $U_{1}, \ldots, U_{n}$ are linked in a cycle, with $U_{i}$ connected to $U_{i+1}$.

Protocol 4. Let $U_{1}, \ldots, U_{n}$ be a dynamic set of users who want to generate a common conference key.

Step $1 \quad$ Each $U_{i}, i=1, \ldots, n$, selects $r_{i} \in_{R} Z_{q}$, and then computes and

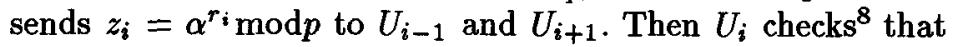
$\operatorname{ord}(\alpha)=q$.

Step 2 Each $U_{i} i=1, \ldots, n$, computes $X_{i} \equiv\left(z_{i+1} / z_{i-1}\right)^{r_{2}}(\bmod p)$.

Let $i=1$. Let $b_{0}=c_{0}=1$. 
Step 3+i-1 $U_{i}$ sends to $U_{i+1}\left(b_{i}, c_{i}\right)$ where $b_{i}=X_{1} \cdot X_{2} \cdots X_{i}(\bmod p)$, and $c_{i}=X_{1}^{i-1} \cdot X_{2}^{i-2} \cdots X_{i-1}(\bmod p)$. Observe that $c_{i}:=$ $b_{i-1} \cdot c_{i-1}(\bmod p)$. Let $i:=i+1$. Let $\ell=1$.

Step $n+1+\ell U_{\ell}$ sends to $U_{\ell+1}: X_{1} \cdot X_{2} \cdots X_{n}(\bmod p)$, and $d_{\ell}=X_{\ell+1}^{n-1}$. $X_{\ell+2}^{n-2} \cdots X_{\ell-1}(\bmod p)$. Observe that $d_{\ell}:=\left(X_{1} \cdot X_{2} \cdots X_{n}\right)$. $d_{\ell-1} \cdot X_{\ell}^{-n}(\bmod p)$.

Step $2 n+2$ Each $U_{i}, i=1, \ldots, n$, computes the conference key,

$$
\begin{aligned}
K_{i} & \equiv\left(z_{i-1}\right)^{n r_{2}} \cdot X_{i}^{n-1} \cdot X_{i+1}^{n-2} \cdots X_{i-2} \\
& \equiv \alpha^{r_{1} r_{2}+r_{2} r_{3}+\cdots+r_{n} r_{1}} \quad(\bmod p) .
\end{aligned}
$$

\subsection{Security}

Theorem 5. If $n$ is polynomially bounded in the length of $p$ and if the DiffieHellman problem is intractable, then Protocols 1,2,3, and \& are conference key distribution systems which guarantee privacy.

\subsection{A Comparison of the Communication and Computational Complexity of the Proposed Systems}

In the following table we summarize the communication and computational costs of the proposed systems (compared to the Diffie-Hellman scheme).

\begin{tabular}{|l|c|c|c|c|c|}
\hline \multicolumn{5}{|c|}{ PRIVACY } & (without authenticity) \\
\hline \multicolumn{1}{|c|}{ Complexity } & Star & Tree & Broadcast & Cyclic \\
\hline \multirow{2}{*}{ Communication } & chair & others & & & \\
Round & $\star 2(n-1)^{\dagger}$ & $\star 1$ & $\star 5^{\ddagger}$ & $\star 2$ & $\star 6$ \\
Computation $^{*}$ & 2 & 2 & $1+\lceil\log n\rceil$ & 2 & $2 n+1$ \\
Delay & $\star n^{\S}$ & $\star 2$ & $\star 4$ & $\star 2+n\lceil\log n\rceil /[\log p\rceil$ & $\star$ constant \\
\cline { 2 - 6 } & \multicolumn{5}{|c|}{ In the final paper } \\
\hline
\end{tabular}

* Per user.

$\dagger$ This means $2(n-1) \star \log p$ bits.

$\ddagger$ Users corresponding to leafs have lower communication and computation costs.

This means $n \star 2 \log p$ multiplications.

Remark. Clearly anybody can masquerade as $U_{i}$ in the protocols described in this section. So the users are not authenticated. In the following section we present an authentication scheme which, when combined with the systems above, offers both privacy and authentication.

\section{Authenticated Conference Key Distribution}

In this section we use a general authentication protocol, e.g. signatures [22]. 
Remark. One has to be careful when using authentication to achieve authenticated key distribution [18]. We discuss this problem and time dependency problems in more details in the final paper.

We are mainly interested in indirect authentication [18].

\subsection{Star Based Authentication}

\section{Protocol 5.}

Each $U_{i}, i=2, \ldots, n$, in Protocol 1 authenticates $z_{i}$ to $U_{1}$, and $U_{1}$ authenticates $z_{1}$ to all $U_{i}$. Then $U_{1}$ sends $Y_{i}$ to each $U_{i}, i=2, \ldots, n$. If some $U_{i}$ fails to authenticate $z_{i}$ then if $i>1, U_{1}$ does not send $Y_{i}$, else $(i=1)$ one stops.

\subsection{Tree Based Authentication}

\section{Protocol 6.}

Each $U_{a}$ in Protocol 2 authenticates $z_{a}$ to its parent $U_{\lfloor a / 2\rfloor}$ and to its sons $U_{2 a}$ and $U_{2 a+1}$ (if these nodes exist). If the authentication of some $z_{a}$ fails no further communication with $U_{a}$ takes place. There is no need to authenticate $Y_{2 a+i}$ if we are only interested in indirect authentication.

A variation of this scheme is obtained by having each $U_{a}$ authenticate $z_{a}$ to its parent in the first round, sequentially (from leafs to root, no parallelism between levels). Then, in the next round, the reverse procedure is used. This idea can be adapted to authenticate the broadcast and cyclic systems (Protocols 3 and 4). Details will be given in the final paper.

\subsection{Broadcast Authentication}

Protocol 7. Each $U_{i}$ in Protocol 3 authenticates $z_{i}$ to $U_{i+1}, i=1, \ldots, n$. If the authentication of $z_{i}$ fails then $U_{i+1}$ halts. Then this process is repeated sequentially. That is, $U_{1}$ first authenticates $z_{1}$ to $U_{2}$. Then each $U_{i}, i=2, \ldots, n$ waits until $z_{i-1}$ is authenticated, and if this is successful, it authenticates the empty string to $U_{i+1}$. This second round serves to guarantee that all the $z_{i}$ are authenticated, as will be explained in the full paper.

\subsection{Cyclic Authentication}

Protocol 8. This is essentially the same as the previous protocol, the only difference being that a cyclic network is used.

\subsection{Security}

Theorem 6. If $n$ is polynomially bounded in the length of $p$ and if the DiffieHellman problem is intractable, and if a secure authentication scheme is used then Protocols 5,6,7, and 8 are conference key distribution systems which are secure against impersonation and substitution attacks. 


\subsection{A Comparison of the Communication and Computational Complexity of the Authenticated Systems}

This is similar to Section 3.6. A table with details is given in the full paper.

\section{An Authentication Scheme}

\subsection{The Basic Scheme}

As in Section 3, a center chooses $p, \alpha$ and $q$, but now $q$ is a prime. Then each user $P$ selects $a, b \in \in_{R} Z_{q}$, computes $\beta=\alpha^{a} \bmod p, \gamma=\alpha^{b} \bmod p$, and registers $k=(\beta, \gamma)$ as its public key. ${ }^{11}$

Protocol 9. Common input: $(p, \alpha, q, \beta, \gamma)$.

$P$ has $a, b$ written on the knowledge tape, where $\beta=\alpha^{a} \bmod p, \gamma=\alpha^{b} \bmod p . P$ is given $z \in Z_{q}$.

$P$ authenticates $z$ to $V: P$ sends $z$ to $V$ and then proves to $V$ that it knows the discrete logarithm of $\beta^{z} \gamma \bmod p(=a z+b \bmod q)$, by using any interactive zero-knowledge proof of knowledge (e.g., $[14,13,3,16])$.

$V$ verifies this and checks ${ }^{8}$ that $\alpha \not \equiv 1(\bmod p), \alpha^{q} \equiv \beta^{q} \equiv \gamma^{q} \equiv 1(\bmod p)$ and that $q$ is a prime. If this fails $V$ halts.

Theorem 7. Protocol 9 is an authentication scheme secure against a generic chosen-message attack $\left(z \in Z_{q}\right.$ is chosen independently of $\left.\gamma\right)$ if the order of $\alpha$ is prime, provided the Discrete Logarithm problem is intractable.

Remark. Although zero-knowledge proofs do not guarantee inherently secure identification [4], in the context of authentication only real-time attacks in which the message is not authentic (e.g., substituted) make sense. To prevent real-time substitution attacks in which the adversary combines proofs of knowledge of different messages, only one proof at a time must be ran. We shall discuss such real-time attacks and ways to avoid them in the full version of the paper.

\subsection{Application to Key Distribution}

Theorem 8. Let $p_{1}, \alpha_{1}$ and $q_{1}$ be as in Section 3, and $p_{2}, \alpha_{2}$ and $q_{2}$ be as in Section 5 with $q_{2}$ a prime and $p_{1} \leq q_{2}$. If each $U_{i}$ authenticates $z_{i}$ as in Protocol 9 with parameters $p_{2}, \alpha_{2}, q_{2}$ and public key $k_{i}=\left(\beta_{2}, \gamma_{2}\right)$, as required in each of the protocols of Section 4, then the conference key distribution systems are secure against impersonation and substitution attacks, provided the DiffieHellman problem is intractable.

Corollary 9. Protocol 9 can be replaced by any proven secure authentication scheme, provided its security assumption is added to the conditions of Theorem 8 .

11 There is no need for $p, q$ to be standard. 


\section{A Proven Secure Authentication Scheme}

The authentication Protocol 9 has not been proven secure against a chosen attack. Indeed in Theorem 7 the proof of security against a substitution attack relies on the independence of the message from $\gamma$, of the public key. We now will modify Protocol 9 to obtain security against all known attacks, including adaptive chosen text attacks.

Let $\left(p_{2}, \alpha_{2}, q_{2}\right),\left(p_{3}, \alpha_{3}, q_{3}\right)$ be as in Section 5 with $p_{2} \leq q_{3}$, and $k=\left(\beta_{2}, \beta_{3}, \gamma_{3}\right)$ be the public key of user $U$, with $\beta_{2}=\alpha_{2}^{a_{2}} \bmod p_{2}, \beta_{3}=\alpha_{3}^{a_{3}} \bmod p_{3}, \gamma_{3}=$ $\alpha_{3}^{b_{3}} \bmod p_{3}, a_{2} \in_{R} Z_{q_{2}}, a_{3}, b_{3} \in \in_{R} Z_{q_{3}}$. The following protocol is used to authenticate any number $z \in Z_{q_{2}}$.

Protocol 10. Common input: $\left(p_{2}, \alpha_{2}, q_{2}, p_{3}, \alpha_{3}, q_{3} ; \beta_{2}, \beta_{3}, \gamma_{3}\right)$.

$P$ has written on its knowledge tape $a_{2}, a_{3}, b_{3}$, where $\beta_{2}=\alpha_{2}^{a_{2}} \bmod p_{2}, \beta_{3}=$ $\alpha_{3}^{a_{3}} \bmod p_{3}, \gamma_{3}=\alpha_{3}^{b_{3}} \bmod p_{3} . P$ is given $z \in Z_{q_{2}}$.

$P$ authenticates $z$ to $V: P$ sends to $V: z$ and $\gamma_{2}=\alpha_{2}^{b_{2}} \bmod p_{2}$, where $b_{2} \in \in_{R} Z_{q_{2}}$, and then proves to $V$, simultaneously, that it knows the discrete logarithm base $\alpha_{2}$ of $\beta_{2}{ }^{z} \cdot \gamma_{2} \bmod p_{2}\left(=a_{2} z+b_{2} \bmod q_{2}\right)$, and the discrete logarithm base $\alpha_{3}$ of $\beta_{3}{ }^{\gamma_{2}} \cdot \gamma_{3} \bmod p_{3}\left(=a_{3} \gamma_{2}+b_{3} \bmod q_{3}\right)$, by using a zero-knowledge proof of knowledge (e.g., $[14,13,3,16])$.

$V$ verifies this, checks that $\gamma_{2}^{q_{2}} \equiv 1\left(\bmod p_{2}\right)$, and then checks ${ }^{8}$ that $\alpha \neq 1(\bmod$ $p), \alpha_{2}^{q_{2}} \equiv \beta_{2}{ }^{q_{2}} \equiv 1\left(\bmod p_{2}\right), \alpha_{3}{ }^{q_{3}} \equiv \beta_{3}{ }^{q_{3}} \equiv \gamma_{3}{ }^{q_{3}} \equiv 1\left(\bmod p_{3}\right)$ and that $q_{2}, q_{3}$ are primes and $p_{2} \leq q_{3}$. If this fails $V$ halts.

Theorem 10. Protocol 9 is a secure authentication scheme if the Discrete Logarithm problem is intractable.

\section{Conclusion}

We have presented a variety of conference key distribution systems which are proven secure against a passive adversary if the Diffie-Hellman problem (a 15 year open problem) is hard. The session key of our main system is a cyclic function (of the indices of the users) of degree two, which is the main reason for its practicality. Ingemarsson Tang and Wong considered conference systems for which the key was a symmetric function of degree two, but these were insecure. Shamir's signature scheme [35], cryptanalyzed by Coppersmith and Stern, also uses symmetric functions. Our results suggest that cyclic functions still have some use in cryptography. Although it is hard for an adversary to compute the session key, it is not clear which bits of this key are hard. Since this problem is also open for the Diffie-Hellman key exchange, it is beyond the scope of this paper.

To achieve security against active adversaries we have extended our conference key distribution protocol. Users have a public key and authenticate their messages using an appropriate authentication scheme. The resulting system is 
proven secure against an active attack under the same assumptions as before, while remaining practical.

The authentication used in our protocol is only proven secure against a generic chosen-message attack [22], i.e., an attack in which the message to be authenticated is chosen independently of the public key (which is sufficient for the security of the conference key system). We have extended our authentication system so that it is also proven secure against an adaptive chosen text attack by a real time middle-person provided the Discrete Logarithm problem is intractable. This resulting scheme remains practical.

\section{Acknowledgements}

The authors wish to thank René Peralta, Adi Shamir, Oded Goldreich, and Moti Yung, for helpful discussions and suggestions, in particular in Section 3.1. Also Kevin McCurley, Tom Berson and Paul van Oorschot for suggestions and various improvements.

\section{References}

1. M. Bellare, S. Goldwasser: New paradigms for digital signatures and message authentication based on non-interactive zero-knowledge proofs. In: G. Brassard, (ed.): Advances in Cryptology - Crypto '89. Lecture Notes in Computer Science 435. Berlin: Springer 1990, pp. 194-211

2. M. Bellare, S. Micali: How to sign given any trapdoor function. Journal of the ACM 39, 214-233 (1992)

3. M. Bellare, S. Micali, R. Ostrovsky: Perfect zero-knowledge in constant rounds. In: Proceedings of the Twenty Second Annual ACM Symp. Theory of Computing. ACM Press 1990, pp. 482-493

4. S. Bengio, G. Brassard, Y.G. Desmedt, C. Goutier, J.-J. Quisquater: Secure implementations of identification systems. Journal of Cryptology 4, pp. 175-183 (1991)

5. C. H. Bennett, G. Brassard: Quantum cryptography, and its application to provable secure key expansion, public-key distribution, and coin tossing. In: International Symposium on Information Theory (abstracts), IEEE Computer Society Press 1983, p. 91

6. R. Blom: Key distribution and key management. In: Proc. Eurocrypt 83, Udine, Italy, March 1983.

7. M. Blum, S. Micali: How to generate cryptographically strong sequences of pseudorandom bits. Siam J. Comput. 13, 850-864 (1984)

8. C. Blundo, A. De Santis, A. Herzberg, S. Kutten, U. Vaccaro, M. Yung: Perfectlysecure key distribution for dynamic conferences. In: E. Brickell (ed.): Advances in Cryptology - Crypto 92. Lecture Notes in Computer Science 740. Berlin: Springer 1993, pp. 471-487

9. J. Boyar, M.W. Krentel, S.A. Kurtz: A discrete logarithm implementation of zeroknowledge blobs. Technical Report 87-002, University of Chicago, March 1987.

10. G. Brassard, D. Chaum, C. Crépeau: Minimum disclosure proofs of knowledge. Journal of Computer and System Sciences 37, 156-189 (1988)

11. M. Burmester: On the risk of opening distributed keys. To appear in the Proceedings of Crypto '94. Berlin: Springer 1994. 
12. J.L. Carter, M.N. Wegman: Universal classes of hash functions. Journal of Computer and System Sciences 18, 143-154 (1979)

13. D. Chaum, J.-H. Evertse, J. van de Graaf: An improved protocol for demonstrating possession of discrete logarithms and some generalizations. In: D. Chaum, W.L. Price (eds.): Advances in Cryptology - Eurocrypt '87. Lecture Notes in Computer Science 304. Berlin: Springer 1988, pp. 127-141

14. D. Chaum, J.-H. Evertse, J. van de Graaf, R. Peralta: Demonstrating possession of a discrete logarithm without revealing it. In: A. Odlyzko (ed.): Advances in Cryptology - Crypto '86. Lecture Notes in Computer Science 263. Berlin: Springer 1987, pp. 200-212

15. D. Coppersmith, A. Odlyzko, R. Schroeppel: Discrete logarithms in $G F(p)$. Algorithmica, pp. 1-15 (1986)

16. Y. Desmedt, M. Burmester: An efficient zero-knowledge scheme for the discrete logarithm based on smooth numbers. In: H. Imai, R.L. Rivest, T. Matsumoto (eds.): Advances in Cryptology - Asiacrypt '91. Lecture Notes in Computer Science 739. Berlin: Springer 1992, pp. 360-367

17. W. Diffie, M. E. Hellman: New directions in cryptography. IEEE Trans. Inform. Theory IT-22, 644-654 (1976)

18. W. Diffie, P.C. van Oorschot, M.J. Wiener: Authentication and authenticated key exchanges. Designs, Codes and Cryptography 2, 107-125 (1992)

19. M. J. Fischer, R. N. Wright: Multiparty secret key exchange using a random deal of cards. In: J. Feigenbaum (ed.): Advances in Cryptology - Crypto '91, Lecture Notes in Computer Science 576. Berlin: Springer 1992, pp. 141-155

20. Z. Galil, S. Haber, M. Yung: A private interactive test of a Boolean predicate and minimum-knowledge public key cryptosystems. In: Annual Symp. on Foundations of Computer Science. IEEE Computer Society Press 1985, pp. 360-371

21. S. Goldwasser, S. Micali, C. Rackoff: The knowledge complexity of interactive proof systems. Siam J. Comput. 18, 186-208 (1989)

22. S. Goldwasser, S. Micali, R. Rivest: A digital signature scheme secure against adaptive chosen-message attacks. Siam J. Comput. 17, 281-308 (1988)

23. D. Gordon: Discrete logarithm in $G F(p)$ using the number field sieve. Submitted.

24. I. Ingemarsson, D.T. Tang, C.K. Wong: A conference key distribution system. IEEE Trans. Inform. Theory 28, 714-720 (1982)

25. K. Koyama, K. Ohta: Identity-based conference key distribution systems. In: C. Pomerance (ed.): Advances in Cryptology - Crypto '87. Lecture Notes in Computer Science 293. Berlin: Springer 1988, pp. 175-185

26. K.S. McCurley: A key distribution system equivalent to factoring. J. Cryptology 1, 95-105 (1988)

27. A. Menezes, S. Vanstone, T. Okamoto: Reducing elliptic curve logarithms to logarithms in a finite field. In: Proceedings of the Twenty Third Annual ACM Symp. Theory of Computing. ACM Press 1991, pp. 80-89

28. M. Naor, M. Yung: Universal one-way hash functions and their cryptographic applications. In: Proceedings of the Twenty First Annual ACM Symp. Theory of Computing. ACM Press 1989, pp. 33-43

29. A.M. Odlyzko: Discrete logs in a finite field and their cryptographic significance. In: N. Cot, T. Beth, I. Ingemarsson, (eds.): Advances in Cryptology - Eurocrypt 84. Lecture Notes in Computer Science 209. Berlin: Springer 1984, pp. 224-314 
30. E. Okamoto: Key distribution systems based on identification information. In: C. Pomerance (ed.): Advances in Cryptology - Crypto '87. Lecture Notes in Computer Science 293. Berlin: Springer 1988, pp. 194-202

31. E. Okamoto, K. Tanaka: Key distribution system based on identification information. IEEE J. Selected Areas in Commun. 7, 481-485 (1989)

32. R.L. Rivest, A. Shamir, L. Adleman: A method for obtaining digital signatures and public key cryptosystems. Commun. ACM 21, 120-126 (1978)

33. J. Rompel: One-way functions are necessary and sufficient for secure signatures. In: Proceedings of the Twenty Second Annual ACM Symp. Theory of Computing. ACM Press 1990, pp. 387-394

34. A. W. Schrift, A. Shamir: The discrete log is very discreet. In: Proceedings of the Twenty Second Annual ACM Symp. Theory of Computing. ACM Press 1990, pp. 405-415

35. A. Shamir: Efficient signature schemes based on birational permutations. To appear in the Proceedings of Crypto '93. Berlin: Springer.

36. S. Tsujii, T. Itoh: An ID-based cryptosystem based on the discrete logarithm. IEEE J. Selected Areas in Commun. 7, 467-473 (1989)

37. M.N. Wegman, J.L. Carter: New hash functions and their use in authentication and set equality. J. Computer and System Sciences 22, 265-279 (1981)

38. Y. Yacobi, Z. Shmuely: On key distribution systems. In: G. Brassard (ed.): Advances in Cryptology - Crypto '89. Lecture Notes in Computer Science 435. Berlin: Springer 1990, pp. 344-355 